(C) 2005 IEEE. Personal use of this material is permitted. Permission from IEEE must be obtained for all other uses, in any current or future media, including reprinting/republishing this material for advertising or promotional purposes, creating new collective works, for resale or redistribution to servers or lists, or reuse of any copyrighted component of this work in other works. 


\title{
Development of a Slotless Tubular Linear Interior Permanent Magnet Micro Motor for Robotic Applications
}

\author{
Haiwei Lu, Jianguo Zhu, Senior Member, IEEE, and Youguang Guo, Member, IEEE
}

\begin{abstract}
Linear micro motors play a key role in micro robotic systems. They can greatly simplify the drive mechanisms, which is crucial for micro systems. By using permanent magnets, much higher force-to-volume ratio can be obtained than using electromagnets and better drive performance can be achieved. This paper describes the development of a tubular permanent magnet linear motor for the actuation of micro robots. Important design criteria are established by both analytical and numerical methods. The field distribution, the electromagnetic force, and the stator phase winding inductances are analyzed and predicted by the finite element analysis taking into account the non-linear properties and saturation effects of the material.
\end{abstract}

Index Terms-Linear motors, linear tubular motors, micro motors, permanent magnet motor, robotics.

\section{INTRODUCTION}

L INEAR MICRO motors play a key role in micro robotic systems. Compared to those pneumatic or rotary-motor based drives, linear motors show significant advantages in terms of efficiency, thrust control, position accuracy, and system volume [1]. Particularly, linear motors can greatly simplify the drive mechanisms, which is crucial for micro systems. By using permanent magnets (PMs) in linear motors, much higher force-to-volume ratio and hence better drive performance than using electromagnets can be obtained.

This paper presents the development of a slotless tubular linear interior permanent magnet (TLIPM) micro motor for drive application in micro robots. Important design criteria are established based on the analytical method under several assumptions and the results are compared with the numerical solutions by the finite element (FE) analysis. The flux density distribution and the electromagnetic force of the motor are analyzed and predicted by the FE analysis. The motor inductances are calculated by considering the non-linear magnetic properties and saturation effects of the material.

The Authors are with the Center for Electric Machines and Power Electronics, Faculty of Engineering, University of Technology, Sydney, PO Box 123, Broadway, NSW 2007, Australia (phone: 61-2-95142337; fax: 612-95142435; e-mail: haiwei@eng.uts.edu.au; jianguo.zhu@uts.edu.au; youguang.guo-1@uts.edu.au).

\section{LINEAR MiCRO MOTOR DESIGN}

\section{A. Motor Structure}

Fig.1 shows the basic configuration of the proposed TLIPM micro motor. The designed external radius $R_{\mathrm{e}}$ of the motor is around $3.5 \mathrm{~mm}$. Three phase windings are mounted inside the stator core. Due to the small external diameter, the slotless structure is selected for simplicity in construction and better performance. The three-phase windings are arranged as $A-C$ $B-A-C-B-A-C-B$ along the axial direction. The material of the PMs used in the micro motor is Nd-Fe-B, and the material for the stator core is the Glassy Metal, an amorphous soft magnetic ribbon material, which features high magnetic permeability and extremely low core loss. The armature shaft supporting the magnets and pole-pieces is made by nonferromagnetic material (i.e. stainless steel) for some advantages such as reduction of the effective air gap between the stator and the armature, the volume of the PM material and the moving mass [2].

\section{B. Optimal Design Considerations}

In order to determine the suitable thickness of the PMs and the diameter of the moving armature, an analytical analysis is firstly performed. Although the linear motor has multiple poles, only one pair of poles is needed to be considered when analyzing the performance of the motor. The magnetic circuit per pole pair used for the analysis is shown in Fig.1.

Under the assumption of infinite magnetic permeability of the iron core and ignoring the leakage flux, by solving the magnetic circuit shown in the figure, the magnetic flux per pole pair $\phi_{g}$ can be worked out as:

$$
\phi_{g}=\mu_{0} \pi H_{c} \frac{\tau_{m}}{4 g /\left(2 l_{m}+2 S+g\right)\left(\tau-\tau_{m}\right)+\tau_{m} / l_{m}\left(l_{m}+2 S\right)}
$$

where

$H_{c}$ coercive force of the PM material;

$\tau$ pole pitch of the armature;

$\tau_{m} \quad$ width of the PM material;

$l_{m} \quad$ radial depth of the PM material;

$g$ the gap between the stator and armature;

$S$ radius of the non-ferromagnetic shaft.

Therefore, the flux density in the air gap $B_{g}$ can be estimated 

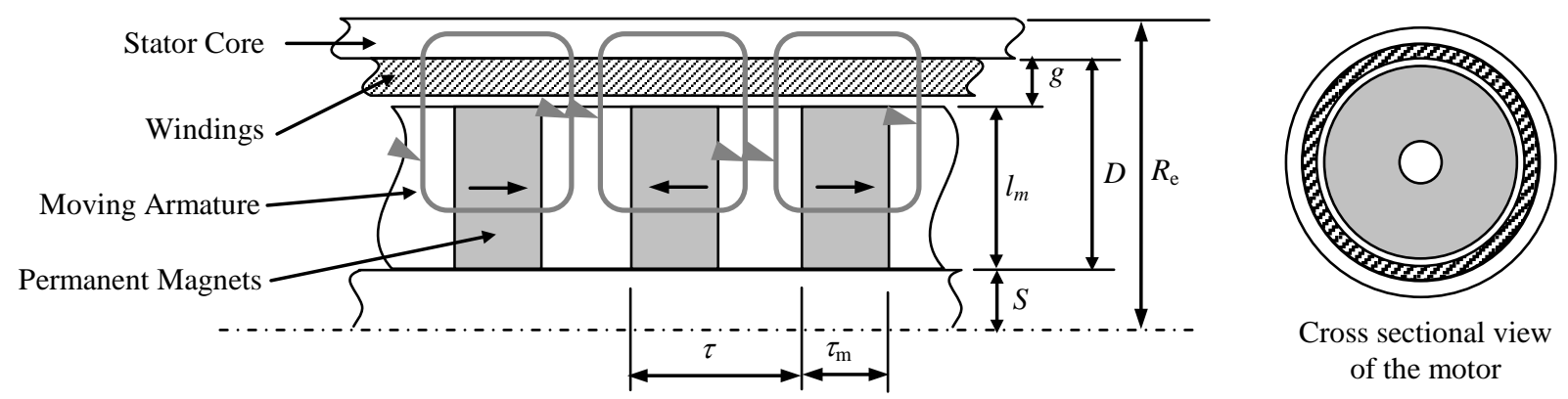

Fig. 1. Configuration of the slotless TLIPM micro motor

by $B_{g}=\phi_{g} / A_{g}$, where $A_{g}$ is the area of the air gap per pole:

$$
B_{g}=2 \mu_{0} H_{c} \frac{\tau_{m}}{4 g+\tau_{m}\left(2 l_{m}+2 S+g\right)\left(\tau-\tau_{m}\right) / l_{m}\left(l_{m}+2 S\right)}
$$

When keeping the volume of the motor and number of poles unchanged, say, taking $l_{m}, g$ and $\tau$ as constants, the flux and the flux density in the air gap will vary with the width of the PMs $\tau_{m}$, as shown in Fig.2. It is seen that there exists a maximum flux at a certain width of PMs. By applying $d \phi_{g} / d \tau_{m}=0$, it can be found that the magnetic flux per pole pair will be maximum when $\tau_{m}=\tau / 2$.

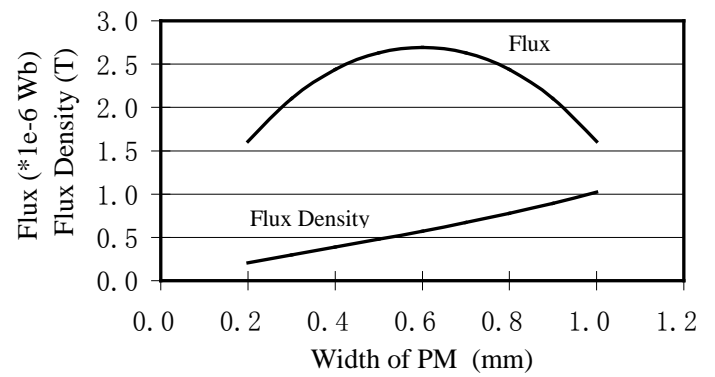

Fig. 2. Flux and flux density in air gap vs. width of PM

The electromagnetic force produced in the stator windings can be worked out by $F=B I L$. Based on the flux density derived in (2) and applying $\tau_{m}=\tau / 2$, the electromagnetic force per pole can be expressed as:

$F_{m}=\mu_{0} \pi H_{c} J_{s} k_{f} \frac{\tau^{2} g l_{m}\left(l_{m}+2 S\right)\left(2 l_{m}+2 S+g\right)}{16 g l_{m}\left(l_{m}+2 S\right)+\tau^{2}\left(2 l_{m}+2 S+g\right)}$

where $J_{s}$ is the current density of the stator windings and $k_{f}$ the fill factor of the windings. If the radii of the motor and the shaft keep constant, or in other words, the gap $D$ between the stator core and the shaft, as shown in Fig.1, is constant, by applying $g=D-l_{m}$, (3) can be rewritten as:

$F_{m}=\mu_{0} \pi H_{c} J_{s} k_{f} \frac{\tau^{2} l_{m}\left(D-l_{m}\right)\left(2 S+l_{m}\right)\left(D+2 S+l_{m}\right)}{16 l_{m}\left(D-l_{m}\right)\left(2 S+l_{m}\right)+\tau^{2}\left(D+2 S+l_{m}\right)}$

From (4), it is shown that the electromagnetic force is a function of $l_{m}$. The variation of the force per pole with $l_{m}$ is computed and is shown in Fig.3 by using the ratio of $l_{m} / D$. It is noticed that there also exists an optimal value of $l_{m}$ which makes the force reach its maximum value at the specified pole pitch and motor radius, and it can be found by applying $d F_{m} / d l_{m}=0$ to (4). For the motor presented in this paper, the optimal ratio of $l_{m} / D$ is found to be 0.778 and the maximum force per pole is $4.6 \mathrm{mN}$ when the current density of the windings $J_{s}$ is set to be $6 \mathrm{~A} / \mathrm{mm}^{2}$.

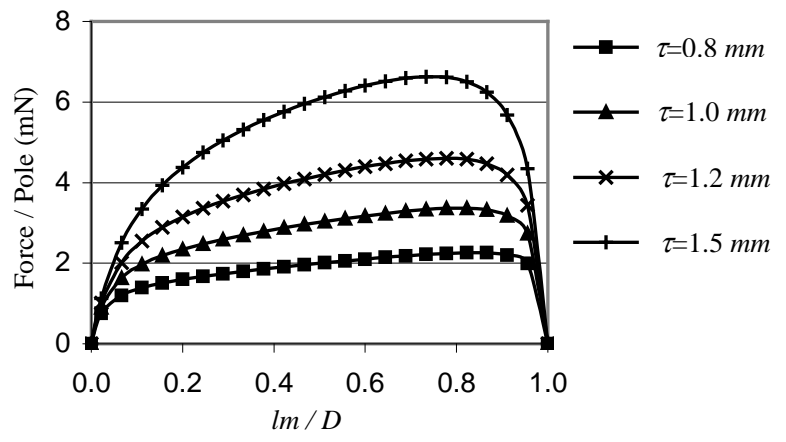

Fig. 3. Electromagnetic force vs. the ratio of $l_{m} / D$

The numerical method is applied to compare with the analytical solution. By a 2D FE analysis, the flux density in the air gap and the electromagnetic forces produced in the stator windings are calculated. When applying $\tau_{m}=\tau / 2$, the effective flux density in the air gap is found to be $0.47 \mathrm{~T}$, which is close to the analytical result. The electromagnetic force is calculated at different $l_{m} / D$ ratios as shown in Fig.4 and the maximum force occurs near $l_{m} / D=0.78$. It is seen that the numerical results agree well with the analytical results.

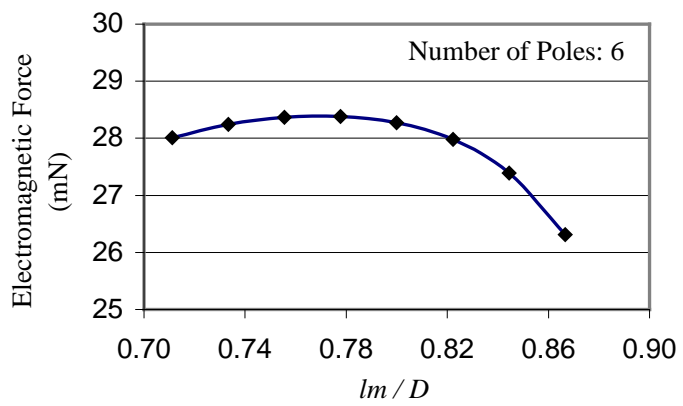

Fig. 4. Electromagnetic force vs. the ratio of $l_{m} / D$ by FE analysis

\section{Motor FluX Density AND ForCE}

Since the moving armature of the micro motor is deliberately designed shorter than the stator so that it can move back and forth within the stator, the distribution of the resultant flux density in the air gap varies with the position of the armature. Consequently, the produced electromagnetic force is also a function of the position of the armature. A 2D 
FE model is established to analyze the flux density in the air gap at different armature positions. Fig.5 shows the flux distribution when the armature is in the middle of the motor. The non-linear properties of magnetic core are considered in the analysis.

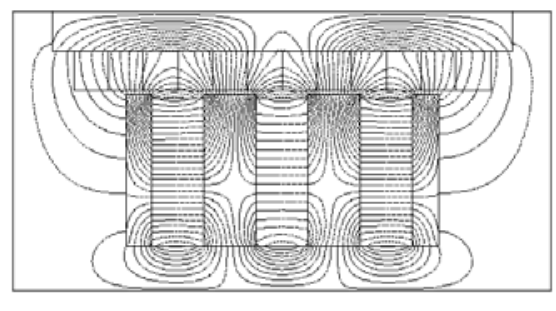

2D TLIPM

Fig. 5. 2D flux distribution in the TLIPM

Based on the analysis of the flux density distribution in the air gap at different armature positions, the produced electromagnetic force can be predicted. The results can provide an optimal current commutation scheme for the motor controller. Fig. 6 shows the motor forces at different armature positions when applying a current density of $6 \mathrm{~A} / \mathrm{mm}^{2}$ and a suitable current switching sequence as $A \bar{B} \rightarrow \bar{B} C \rightarrow \bar{A} C \rightarrow \bar{A} B$. The three positions which generate the lowest forces will be used as the current commutation points.

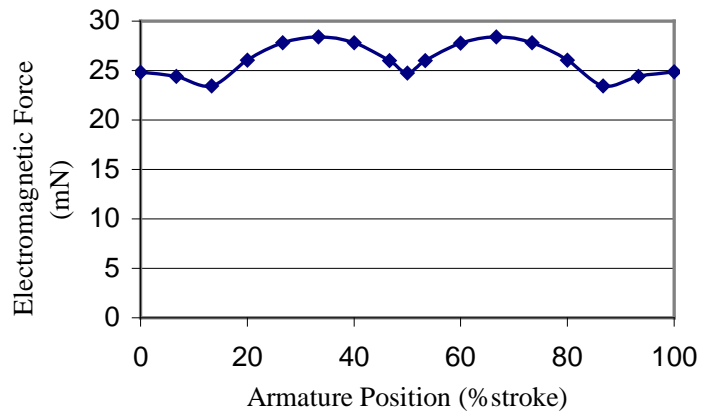

Fig. 6. Motor force vs. armature positions

\section{STATOR WINDING INDUCTANCES}

The stator winding inductance is one of the most important parameters for the motor modeling, performance simulation and controller design. In this paper, the energy method is applied for the inductance computation. However, due to the non-linear properties of the magnetic core, it is difficult to use 2D FE model to work out the stored magnetic energy in such a tubular structure. Thus, a 3D FE model is applied and during the analysis, both the non-linear and saturation effects are considered to improve the accuracy of calculation.

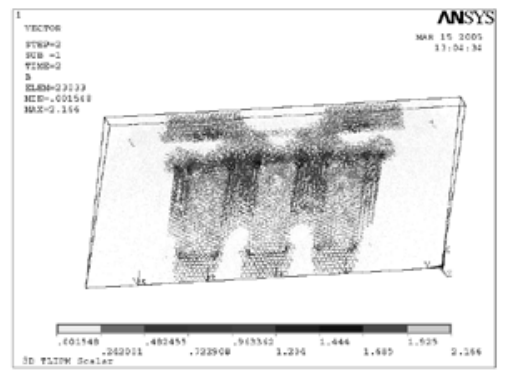

Fig. 7. 3D FE model of TLIPM with flux density distribution
Because of the non-linear properties of the Glassy Metal, it is necessary to find out the operation point set by the PMs before the magnetic energy produced by the stator current can be correctly obtained. Fig.7 shows the flux density produced only by the PMs via a 3D non-linear FE model. Based on the results, the magnetic flux density in each part of the magnetic core of the motor can be obtained and the related magnetic permeability can be found. Table I lists the relative permeability in different magnetic core regions.

TABLE I Relative permeability in different motor parts

\begin{tabular}{|c|c|c|c|c|}
\hline $\begin{array}{c}\text { Armature } \\
\text { core } \\
\text { Upper }\end{array}$ & $\begin{array}{c}\text { Armature } \\
\text { core } \\
2^{\text {nd }} \text { layer }\end{array}$ & $\begin{array}{c}\text { Armature } \\
\text { core } \\
3^{\text {rd }} \text { layer }\end{array}$ & $\begin{array}{c}\text { Armature } \\
\text { core } \\
\text { Bottom }\end{array}$ & $\begin{array}{c}\text { Stator } \\
\text { core }\end{array}$ \\
\hline 258 & 121 & 121 & 275 & 478400 \\
\hline
\end{tabular}

From Table I, it is shown that the armature core is saturated, especially the pole-pieces in the middle of the armature. Taking the results as the operation point, the magnetic energy generated by the stator winding current is calculated by ignoring the PMs, and the inductances of the windings can then be worked out by the energy method. The computational results at different armature positions are shown in Table II. It is seen that inductances will not change much at different armature positions.

TABLE II Stator winding self and mutual inductances (Unit: $\mu \mathrm{H}$ )

\begin{tabular}{|c|c|c|c|c|c|c|}
\hline Position & $L_{\mathrm{a}}$ & $L_{\mathrm{b}}$ & $L_{\mathrm{c}}$ & $L_{\mathrm{ab}}$ & $L_{\mathrm{bc}}$ & $L_{\mathrm{ac}}$ \\
\hline 0\% stroke & 15.0 & 15.0 & 14.8 & 2.43 & 7.59 & 7.62 \\
\hline 50\% stroke & 14.8 & 14.8 & 15.2 & 3.06 & 7.37 & 7.37 \\
\hline 100\% stroke & 15.0 & 15.0 & 14.9 & 2.43 & 7.62 & 7.58 \\
\hline
\end{tabular}

It is noted that the mutual inductance between phases $\mathrm{A}$ and $\mathrm{B}, L_{\mathrm{ab}}$, is much smaller than the other two mutual inductances. This is due to the unsymmetrical distribution of the phase windings, which makes the coupling effect between phase A and $\mathrm{B}$ is less than that of others.

\section{CONCLUSION}

A PM linear motor has been developed for linear motion micro robotic systems. Important design criteria are established by the analytical study of the flux and electromagnetic force of the motor. Numerical solutions are applied to compare with the analytical analysis and the results show a good consistency. The force of the micro motor are predicted at different armature positions based on the FEA and an optimal commutation scheme is obtained for the motor controller. The results show that the proposed micro motor has a satisfactory force capability. In addition, the inductances of the motor are calculated by considering the non-linear properties and saturation effects of the material, which will be very helpful to the design of the motor controller.

\section{REFERENCES}

[1] N. Bianchi, S. Bolognani, F. Tonel, "Design Consideration for a Tubular Linear PM Servo Motor,” EPE Journal, vol. 11, no. 3, Aug. 2001, pp. 41-47.

[2] J. Wang, D. Howe, G. W. Jewell, "Analysis and Design Optimization of an Improved Axially Magnetized Tubular Permanent-Magnet Machine," IEEE Trans. Energy Conversion, vol. 19, no.2, June, 2004, pp. 289-295.

[3] B. Lequesne, "Permanent magnet linear motors for short strokes," IEEE Trans. Ind. Applicat., vol. 32, Issue 1, Jan./Feb. 1996, pp. 161-168. 